\title{
EXPLORE: A Prospective, Multinational, Natural History Study of Patients with Acute Hepatic Porphyria with Recurrent Attacks
}

\author{
Laurent Gouya, ${ }^{1-3}$ Paolo Ventura, ${ }^{4 *}$ Manisha Balwani (DD ${ }^{5 *}$ D. Montgomery Bissell, ${ }^{6}$ David C. Rees, ${ }^{7}$ Ulrich Stölzel, ${ }^{8}$ \\ John D. Phillips, ${ }^{9}$ Raili Kauppinen, ${ }^{10}$ Janneke G. Langendonk, ${ }^{11}$ Robert J. Desnick, ${ }^{5}$ Jean-Charles Deybach, ${ }^{1-3}$ \\ Herbert L. Bonkovsky (D) ${ }^{12}$ Charles Parker, ${ }^{9}$ Hetanshi Naik, ${ }^{5}$ Michael Badminton, ${ }^{13}$ Penelope E. Stein, ${ }^{7}$ Elisabeth Minder, ${ }^{14}$ \\ Jerzy Windyga, ${ }^{15}$ Radan Bruha, ${ }^{16}$ Maria Domenica Cappellini, ${ }^{17}$ Eliane Sardh, ${ }^{18}$ Pauline Harper, ${ }^{18}$ Sverre Sandberg, ${ }^{19}$ \\ Aasne K. Aarsand, ${ }^{19}$ Janice Andersen, ${ }^{19}$ Félix Alegre, ${ }^{20}$ Aneta Ivanova, ${ }^{21}$ Neila Talbi, ${ }^{1-3}$ Amy Chan, ${ }^{22}$ William Querbes, ${ }^{22}$ John Ko, ${ }^{22}$ \\ Craig Penz, ${ }^{22}$ Shangbin Liu, ${ }^{22}$ Tim Lin, ${ }^{22}$ Amy Simon, ${ }^{22}$ and Karl E. Anderson ${ }^{23}$
}

BACKGROUND AND AIMS: Acute hepatic porphyria comprises a group of rare genetic diseases caused by mutations in genes involved in heme biosynthesis. Patients can experience acute neurovisceral attacks, debilitating chronic symptoms, and long-term complications. There is a lack of multinational, prospective data characterizing the disease and current treatment practices in severely affected patients.

APPROACH AND RESULTS: EXPLORE is a prospective, multinational, natural history study characterizing disease activity and clinical management in patients with acute hepatic porphyria who experience recurrent attacks. Eligible patients had a confirmed acute hepatic porphyria diagnosis and had experienced $\geq 3$ attacks in the prior 12 months or were receiving prophylactic treatment. A total of 112 patients were enrolled and followed for at least 6 months. In the 12 months before the study, patients reported a median (range) of 6 (0-52) acute attacks, with $52(46 \%)$ patients receiving hemin prophylaxis. Chronic symptoms were reported by 73 (65\%) patients, with $52(46 \%)$ patients experiencing these daily. During the study, 98 (88\%) patients experienced a total of
483 attacks, $77 \%$ of which required treatment at a health care facility and/or hemin administration (median [range] annualized attack rate 2.0 [0.0-37.0]). Elevated levels of hepatic $\delta$-aminolevulinic acid synthase 1 messenger ribonucleic acid levels, $\delta$-aminolevulinic acid, and porphobilinogen compared with the upper limit of normal in healthy individuals were observed at baseline and increased further during attacks. Patients had impaired quality of life and increased health care utilization.

CONCLUSIONS: Patients experienced attacks often requiring treatment in a health care facility and/or with hemin, as well as chronic symptoms that adversely influenced day-to-day functioning. In this patient group, the high disease burden and diminished quality of life highlight the need for novel therapies. (Hepatology 2019;0:1-13).

$\Lambda$ cute hepatic porphyria (AHP) comprises a group of rare metabolic diseases caused by mutations in genes encoding enzymes involved in heme biosynthesis. ${ }^{(1,2)}$ The four types

Abbreviations: $A A R$, annualized attack rate; $A H P$, acute hepatic porphyria; $A I P$, acute intermittent porphyria; $A L A, \delta$-aminolevulinic acid; $A L A S 1$, $\delta$-aminolevulinic acid synthase 1; CKD, chronic kidney disease; eGFR, estimated glomerular filtration rate; EQ-5D-5L, EuroQoL 5-dimensions questionnaire 5-levels; HCP, hereditary coproporphyria; LC-MS/MS, liquid chromatography-tandem mass spectrometry; PBG, porphobilinogen; QoL, quality of life; ULN, upper limit of normal; VP, variegate porphyria.

Received May 10, 2019; accepted September 4, 2019.

Additional Supporting Information may be found at onlinelibrary.wiley.com/doi/10.1002/hep.30936/suppinfo.

*These authors are equally contributors to this work.

(C) 2019 The Authors. HEPATOLOGY published by Wiley Periodicals, Inc., on behalf of American Association for the Study of Liver Diseases. This is an open access article under the terms of the Creative Commons Attribution-NonCommercial License, which permits use, distribution and reproduction in any medium, provided the original work is properly cited and is not used for commercial purposes.

View this article online at wileyonlinelibrary.com.

DOI 10.1002/hep.30936 
of AHP are autosomal dominant acute intermittent porphyria (AIP), variegate porphyria (VP), hereditary coproporphyria $(\mathrm{HCP})$, and the extremely rare autosomal recessive $\delta$-aminolevulinic acid (ALA) dehydratase deficient porphyria. ${ }^{(3,4)}$ AIP is the most common type, with a prevalence of disease-related mutations of approximately 1:1,700, with penetrance estimated at approximately $20 \%$ in families with AIP but only $1 \%$ in the general population..$^{(5,6)}$

Induction of the heme synthesis pathway in the liver through the initial and rate-limiting enzyme $\delta$-aminolevulinic acid synthase 1 (ALAS1) can be precipitated by a variety of triggers. In patients with AHP, this induction can result in the accumulation of toxic heme intermediates, primarily ALA and porphobilinogen $(\mathrm{PBG})$, which are likely responsible for disease manifestations that include potentially life-threatening neurovisceral attacks. ${ }^{(7-9)}$

During attacks, patients typically present with multiple signs and symptoms caused by dysfunction across the autonomic, central, and peripheral nervous systems. They often require hospitalization and pain relief with opioids. ${ }^{(7,9)}$ Most symptomatic patients have only a few attacks in their lifetimes; however, approximately $3 \%-8 \%$ of symptomatic patients with AIP experience recurrent attacks. ${ }^{(10,11)}$ Patients may also experience chronic symptoms between attacks, ${ }^{(12-14)}$ a negative impact on their physical and emotional health, ${ }^{(4,12)}$ and diminished quality of life (QoL). ${ }^{(15)}$ In addition, long-term complications related to AHP and its treatment can occur, including liver disease (e.g., cirrhosis and hepatocellular carcinoma), ${ }^{(16,17)}$ chronic kidney disease (CKD; as assessed by estimated glomerular filtration rate $[\mathrm{eGFR}])$, and systemic arterial hypertension. ${ }^{(9,18-20)}$

Given the rarity of the disease and the nonspecific constellation of signs and symptoms, patients typically experience substantial delays in diagnosis, with one study suggesting a mean time to diagnosis of 15 years from symptom onset. ${ }^{(13)}$ This is of concern,

Potential conflict of interest: Dr. Gouya received grants from Alnylam. Dr. Ventura consults for, advises, and received grants from Alnylam. He consults for and received grants from Recordati. Dr. Balwani consults for and received grants from Alnylam. She consults for Recordati. Dr. Rees consults and is on the speakers' bureau for Alnylam. Dr. Kauppinen owns stock in Orion. Dr. Bonkovsky consults for and received grants from Alnylam. He consults for Recordati. Dr. Desnick consults for and received grants from Alnylam and Recordati. Dr. Deybach consults for Alnylam. Dr. Naik consults for and advises Alnylam. Dr. Stein received grants from Alnylam. Dr. Harper received grants from Alnylam. Dr. Minder consults for Alnylam. Dr. Sardh received grants from Alnylam. Dr. Windyga received grants from Alnylam. Dr. Anderson consults for, advises, and received grants from Alnylam, Recordati, and Mitsubishi. Dr. Chan is employed by and owns stock in Alnylam. Dr. Ko is employed by and owns stock in Alnylam. Dr. Liu is employed by and owns stock in Alnylam. Dr. Penz is employed by and owns stock in Alnylam. Dr. Querbes owns stock in Alnylam. Dr. Sandberg advises Alnylam. Dr. Simon is employed by and owns stock in Alnylam.

\section{ARTICLE INFORMATION:}

From the ${ }^{1}$ Centre de Référence Maladies Rares Porphyries, Colombes, France; ${ }^{2}$ University of Paris, Paris, France; ${ }^{3}$ Laboratory of Excellence GR-Ex, Paris, France; ${ }^{4}$ Università degli Studi di Modena e Reggio Emilia, Emilia-Romagna, Italy; ${ }^{5}$ Icahn School of Medicine at Mount Sinai, New York, NY; ${ }^{6}$ University of California, San Francisco, CA; ${ }^{7}$ King's College Hospital, King's College London, London, UK; ${ }^{8}$ Klinikum Chemnitz Porphyria Center, Chemnitz, Germany; ${ }^{9}$ University of Utah, Salt Lake City, UT; ${ }^{10}$ University Hospital of Helsinki, Helsinki, Finland; ${ }^{11}$ Porphyria Center, Center for Lysosomal and Metabolic Disease, Department of Internal Medicine, Erasmus MC, University Medical Center Rotterdam, the Netherlands; ${ }^{12}$ Section on Gastroenterology \& Hepatology, Wake Forest University/NC Baptist Medical Center, Winston-Salem, NC; ${ }^{13}$ University Hospital of Wales, Cardiff, UK; ${ }^{14}$ Stadtspital Triemli, Zentrallabor, Zurich, Switzerland; ${ }^{15}$ Department of Hemostatic Disorders and Internal Medicine, Institute of Hematology and Transfusion Medicine, Warsaw, Poland; ${ }^{16} 4$ th Internal Clinic, General University Hospital, Charles University, Prague, Czech Republic; ${ }^{17}$ Fondazione IRCCS, Ca Granda, University of Milan, Milan, Italy; ${ }^{18}$ Porphyria Centre Sweden, Centre for Inherited Metabolic Diseases, Karolinska Institutet, Karolinska University Hospital, Stockholm, Sweden; ${ }^{19}$ Norwegian Porphyria Centre, Haukeland University Hospital, Bergen, Norway; ${ }^{20}$ Clinica Universidad de Navarra, Navarra, Spain; ${ }^{21}$ St. Ivan Rilski U Hospital, Sofia, Bulgaria; ${ }^{22}$ Alnylam Pharmaceuticals, Cambridge, MA; ${ }^{23}$ University of Texas Medical Branch, Galveston, TX.

\section{ADDRESS CORRESPONDENCE AND REPRINT REQUESTS TO:}

Laurent Gouya, M.D., Ph.D.

Centre de Référence Maladies Rares Porphyries Service de Biochimie Métabolisme et Nutrition CHU Paris Nord-Val de Seine - Hôpital Louis Mourier
178 rue des Renouillers 92700 Colombes, France

E-mail: laurent.gouya@inserm.fr

Tel.: +1-33- (0)1-47606334 
as patients may experience worsening disease manifestations while undergoing diagnostic testing or treatment for an incorrect diagnosis. Attacks that are not treated promptly can lead to severe neurologic damage, require prolonged periods of recovery, or, in some cases, are fatal. ${ }^{(9,21,22)}$

The only approved treatment for acute attacks is hemin (heme arginate in Europe and lyophilized hematin in the United States), which is administered intravenously and acts by down-regulating hepatic ALAS1. ${ }^{(23,24)}$ There are no approved pharmacotherapies for attack prevention. ${ }^{(25)}$ Current strategies include trigger avoidance (e.g., porphyrinogenic drugs, fasting), early ingestion of carbohydrates, suppression of ovulation, and off-label intravenous hemin prophylaxis. ${ }^{(7,26)}$ However, prophylactic hemin may induce dependence on exogenous heme, has potential acute side effects (e.g., infusion site reactions, nausea, fatigue, and headache), and may result in potential complications related to chronic administration (e.g., iron overload, tachyphylaxis, and venous obliteration). ${ }^{(7,11,18,26)}$ Hormonal interventions to prevent ovulation include gonadotropin-releasing hormone analogs with or without add-back estrogen and lowdose oral contraceptives ${ }^{(27-29)}$ but are complex and applicable only in select patients. In a small number of severely affected patients, liver transplantation has resulted in normalization of ALA and PBG and the cessation of porphyria attacks. ${ }^{(30)}$ Limitations include a shortage of donors, highly invasive operations, lifelong immunosuppression therapy, and high rates of hepatic artery thrombosis. ${ }^{(30)}$ Thus, there is a high unmet medical need for effective treatments.

Addressing these needs is impaired by a limited understanding of the natural history of AHP. Although there are a few natural history studies characterizing patients with AHP and their management, ${ }^{(13,31,32)}$ there have been no multinational, prospective, longitudinal studies that document the burden of disease in severely affected patients who experience recurrent attacks. The present study, EXPLORE, was thus designed to characterize the natural history and current clinical management of patients with AHP experiencing recurrent attacks. Secondary objectives included characterization of levels of urinary and serum ALA, PBG, and circulating hepatic ALAS1 mRNA levels during and between attacks; AHP signs and symptoms during and between attacks; medical and medication history; familial history; QoL; and health care utilization.

\section{Patients and Methods}

\section{STUDY DESIGN AND OVERSIGHT}

EXPLORE is a prospective, multinational, observational, natural history study in patients with $\mathrm{AHP}$ experiencing recurrent attacks (defined in this study as $\geq 3$ porphyria attacks within 12 months before the baseline visit) or on prophylactic treatment to prevent attacks. The study was initiated in September 2014, completed in November 2016, and conducted at 21 centers ( 7 in the United States and 14 in Europe). The study included clinical visits (with physical examination, patient questionnaires, blood draws, and laboratory assessments) at baseline, 6 months, and an optional visit at 12 months, with telephone follow-up every 2 months (Supporting Table S1). The study design was developed in collaboration with the sponsor, principal investigators, and the American Porphyria Foundation. All patients provided informed consent in writing. The study was approved by central and local institutional review boards or ethics committees according to the International Conference on Harmonization for Good Clinical Practice and the World Health Organization Declaration of Helsinki. The trial is registered at www.ClinicalTr ials.gov (NCT02240784).

\section{PATIENTS}

Male or female patients aged $\geq 18$ years were eligible if they had a diagnosis of AIP, HCP, or VP made by a porphyria specialist. Diagnosis was based on a history of clinical manifestations of AHP (such as acute attacks of abdominal, back, or limb pain), biochemical evidence of an AHP attack $(\geq 1$ documented urine or plasma PBG level $>4$ times the upper reference limit), and presence of a pathogenic genetic mutation. If no mutation had been identified by sequencing of relevant heme biosynthesis genes, patients were eligible if they had an AIP, HCP, or VP diagnosis made by a porphyria specialist, documented PBG elevation, and either decreased hydroxymethylbilane synthase activity in erythrocytes or increased fecal coproporphyrins with a ratio of coproporphyrin III/coproporphyrin I >4 with or without elevation of fecal protoporphyrin.

Eligible patients had to be experiencing recurrent attacks defined by meeting one of the following: $\geq 3$ 
porphyria attacks within 12 months before the baseline visit, monthly (or more frequent) prophylactic hemin treatment (regardless of attack frequency), or prophylactic gonadotropin-releasing hormone ana$\log$ treatment (regardless of attack frequency). At least one of the prior attacks had to require hemin or treatment at a hospital or in a health care setting (e.g., clinic or infusion center). Patients were excluded if they were actively participating in a clinical trial of an investigational product. Patients could also be excluded if they were not considered a good candidate for participation as judged by a study investigator.

\section{STUDY ASSESSMENTS}

Full details of study assessments are in Supporting Table S1. In brief, at clinic visits (baseline, Month 6, and optional Month 12), patients completed questionnaires detailing AHP symptoms and treatment, QoL (using EuroQoL 5-dimensions questionnaire 5-levels [EQ-5D-5L], a QoL measure used in multiple diseases and population groups $\left.{ }^{(33)}\right)$, and health status. Physical examinations and laboratory assessments (including urinary porphyrin precursor analysis, liver function tests, hematology, and serum chemistry) were also performed, with all samples being shipped on dry ice (for sample collection at sites) or freezer packs (for home sample collection) to a central laboratory for analysis. Spot urine ALA and PBG were assessed by a validated assay at a central laboratory (Covance Laboratory, Utah) using liquid chromatography-tandem mass spectrometry (LC-MS/MS) ${ }^{(34)}$ and results normalized to urine creatinine. The upper reference limit for urinary ALA and PBG was established by assessing urinary ALA and PBG levels in 150 normal healthy subjects. ${ }^{(35)}$ Circulating hepatic ALAS1 mRNA levels (hereafter called ALAS1 mRNA) were measured in exosomes isolated from spot urine samples using the circulating extracellular RNA detection assay (as described ${ }^{(36)}$ ). ALAS1 mRNA levels are expressed relative to normal healthy levels. ${ }^{(36)}$ For remote assessments, performed at Months 2 and 4 and optional Months 8 and 10, patients completed questionnaires detailing AHP symptoms and treatment and mailed a spot urine sample to assess PBG and ALA levels. Any adverse medical occurrence in a patient that was judged by the investigator as not related to a study procedure was recorded as a change in health status.

\section{DEFINITION AND ASSESSMENT OF AHP ATTACKS}

An AHP attack was reported by the patient if the attack required increased pain medication or carbohydrate intake, hemin administration, and/ or hospitalization for signs and symptoms of AHP, including but not limited to severe abdominal pain, vomiting, tachycardia, constipation, arterial hypertension, or hyponatremia. An attack was defined as new if it started at least 1 calendar day after the last attack ended. Attacks were classified according to the duration and location of attack treatment (e.g., home or health care facility). The frequency of AHP attacks was assessed by calculating the mean annualized attack rate (AAR).

During an attack, urine ALA and PBG levels were measured daily, and, to capture symptoms experienced, patients completed an attack symptom inventory form daily. If the attack required hospitalization, then physical and neurologic examinations, vital signs, available laboratory assessments, and attack treatment were recorded. One and 2 weeks after an attack, ongoing AHP symptoms were captured during phone interviews conducted by study center staff.

\section{STATISTICAL ANALYSIS}

Data from all patients who completed screening were analyzed. As EXPLORE is an observational study, no formal hypothesis testing was conducted, and sample size was not based on statistical considerations. Results were analyzed using descriptive statistics.

\section{Results}

\section{STUDY POPULATION}

A total of 112 patients were enrolled, 104 of whom had AIP, 3 had HCP, and 5 had VP (Table 1). Patients were predominantly female (89\%) and Caucasian (85\%), with 56\% from Europe and $44 \%$ from the United States. Ninety-six (86\%) patients had genotype results available, which identified 64 distinct mutations in the bydroxymethylbilane 
TABLE 1. Patient Demographics and Disease Characteristics in EXPLORE

\begin{tabular}{|c|c|}
\hline Variable & Patients $(n=112)$ \\
\hline Age in years & $38(19-70)$ \\
\hline Female sex & $100(89)$ \\
\hline \multicolumn{2}{|l|}{ Ethnicity } \\
\hline White & $95(85)$ \\
\hline Asian & $3(3)$ \\
\hline Black & $3(3)$ \\
\hline Not provided & $11(10)$ \\
\hline \multicolumn{2}{|l|}{ Geographic region } \\
\hline Europe & $63(56)$ \\
\hline United States & $49(44)$ \\
\hline \multicolumn{2}{|l|}{ AHP type } \\
\hline AIP & $104(93)$ \\
\hline $\mathrm{HCP}$ & $3(3)$ \\
\hline VP & $5(4)$ \\
\hline Relative with known or suspected AHP & $83(74)$ \\
\hline Time since first attack in years & $8.0(0.5-41.1)$ \\
\hline Number of attacks in the last 12 months & $6(0-52)$ \\
\hline $\begin{array}{l}\text { Number of attacks in the last } 12 \text { months requiring } \\
\text { hemin treatment and/or hospitalization }\end{array}$ & $4(0-50)$ \\
\hline \multicolumn{2}{|l|}{$\begin{array}{l}\text { Number of patients reporting attacks in the last } \\
12 \text { months }\end{array}$} \\
\hline 0 attacks & $6(5)$ \\
\hline 1-2 attacks & $6(5)$ \\
\hline 3-5 attacks & $42(38)$ \\
\hline 6-10 attacks & $22(20)$ \\
\hline$>10$ attacks & $36(32)$ \\
\hline Patients experiencing chronic AHP symptoms & $73(65)$ \\
\hline $\begin{array}{l}\text { Patients who received prior hemin treatment for } \\
\text { attacks }\end{array}$ & $94(84)$ \\
\hline Current hemin prophylaxis & $52(46)$ \\
\hline Weekly dosing & $22(42)$ \\
\hline Monthly dosing & $10(19)$ \\
\hline Unknown dosing & $4(8)$ \\
\hline Other & $16(31)$ \\
\hline Time in years on hemin prophylaxis & $5(0-26)$ \\
\hline
\end{tabular}

Note: Data are presented as n (\%) or median (range).

synthase gene in patients with AIP. The most common genotypes were p. R173W (4 [4\%] patients) and p. W283X (4 [4\%] patients). The 8 patients with VP and HCP all had distinct genotypes. Six-month and optional 12-month follow-up visits were completed by 107 (96\%) and 80 (71\%) patients, respectively (Supporting Fig. S1), and the mean (standard deviation [SD]) of duration on study was 10.6 (2.8) months. There were no deaths during the study, but one death was reported by an investigator in a patient after she had left the study (see On-Study Changes in Health Status).

\section{DISEASE AND TREATMENT CHARACTERISTICS AT BASELINE}

In the 12 months before study entry, patients reported a median (range) of 6 (0-52) attacks, with $32 \%$ experiencing $>10$ attacks (Table 1 ). Of those attacks, the median number (range) that required hemin or treatment at a health care facility was 4 (0-50). Patients reported a broad array of signs and symptoms associated with attacks (Fig. 1A). Almost all patients (99\%) reported pain during attacks, mostly abdominal pain (92\%), arm/leg pain (77\%), and back pain (72\%). Other common signs and symptoms (occurring in $>80 \%$ of patients) included nausea (85\%) and change in urine color (81\%; Fig. 1A). Chronic symptoms occurring between attacks were reported by $73(65 \%)$ patients (Fig. 1B), with 52 (46\%) patients experiencing these daily. The most common chronic symptoms were pain, nausea, tiredness, and anxiety.

Before entering the study, hemin treatment for attacks had been received by 94 (84\%) patients, with side effects reported in 55 (59\% of those who had received hemin) patients. Common side effects reported because of hemin use included headache, flu-like symptoms, increased iron or ferritin levels (secondary iron overload), phlebitis, thrombosis, and difficulties with venous access. Sixty-two (55\%) patients reported taking medications prophylactically for AHP at baseline, of whom 52 (46\%) patients received hemin (Table 1), $6 \%$ were on a gonadotropin-releasing hormone analog, and $22 \%$ took "other" medications (including opioids, nonsteroidal anti-inflammatory drugs, antidepressants, and benzodiazepines, among others). The most common regimen reported for those on hemin prophylaxis was weekly (42\% of patients). Rates of hemin prophylaxis were slightly higher in the United States (53\% of patients) than in Europe ( $41 \%$ of patients). Chronic symptoms were reported in a similar number of AIP patients who received hemin prophylaxis $(n=33$ [66\%]) as those who received no hemin prophylaxis $(n=34[63 \%])$ before screening.

\section{CONCOMITANT MEDICAL HISTORY}

Medical history or conditions commonly associated with AHP included placement of a central venous catheter in 45 (40\%) patients, systemic arterial hypertension in 27 (24\%) patients, and depression in 20 (18\%) patients (Supporting Table S2). In addition, 

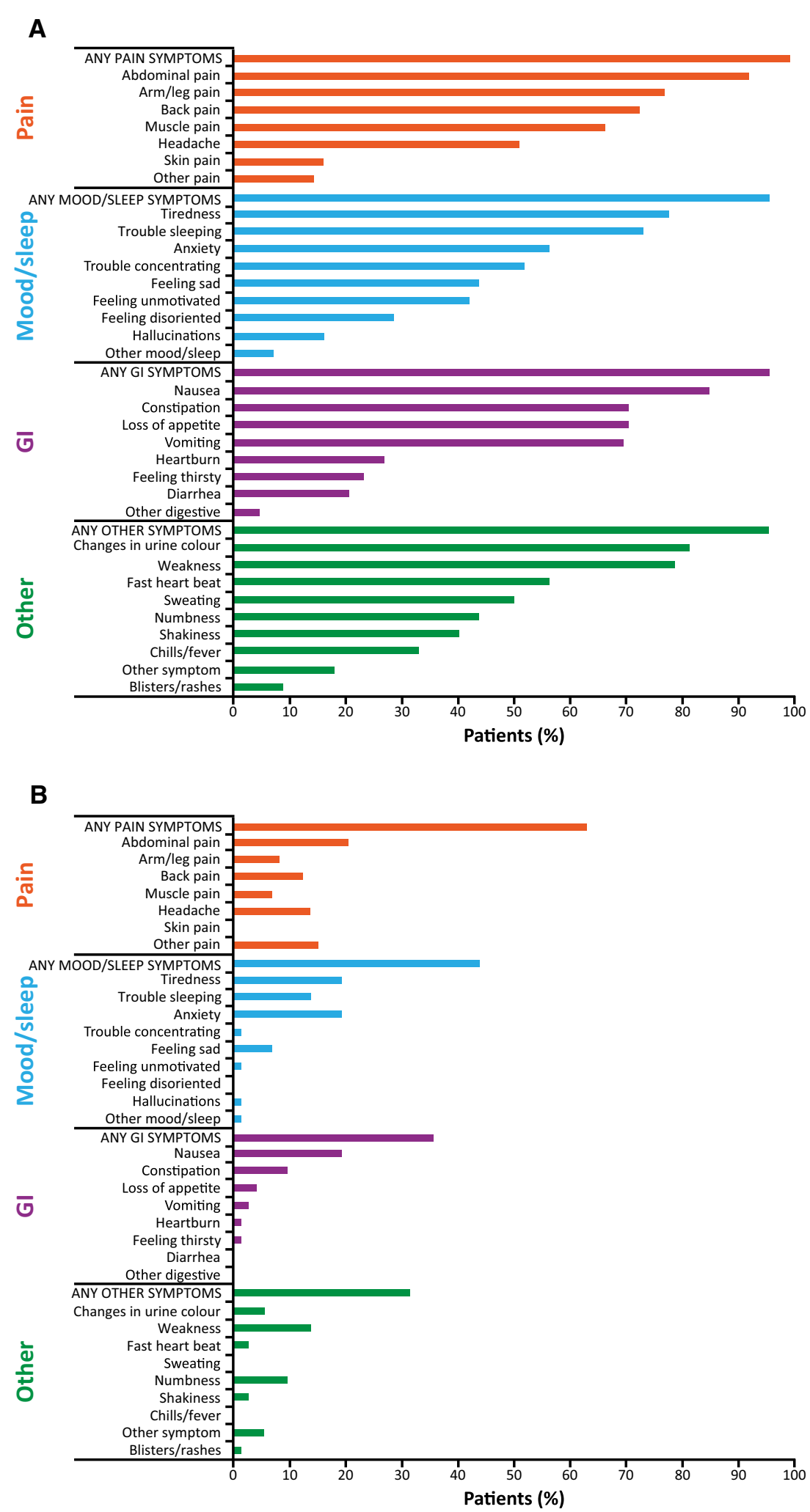

FIG. 1. Percentage of patients reporting attack or chronic symptoms at baseline. (A) Attack symptoms and (B) chronic symptoms. Symptom categories (pain, mood/sleep, GI, and other) were used when collecting data from patients to aid the reporting process. Chronic symptoms between attacks were reported by 73 (65\%) patients; percentages are based on these 73 patients. Abbreviation: GI, gastrointestinal. 
12 (11\%) patients experienced renal or urinary disorders, including 5 (4\%) patients with renal failure and an additional 3 (3\%) patients with CKD, based on medical history. Three (3\%) patients had a history of infection related to a central venous catheter and $5(4 \%)$ patients had experienced thrombotic events related to a central catheter. All patients reported taking at least one prior and ongoing medication at the time of study entry. The most common medications were those used to treat or prevent AHP symptoms, including hemin in 83 (74\%) patients, natural opium alkaloids in $62(55 \%)$ patients, parenteral glucose solutions in 37 (33\%) patients, and extra oral carbohydrates in $30(27 \%)$ patients. The most frequently reported other porphyria-related medications were paracetamol (25\%), ondansetron (22\%), gabapentin (13\%), and lorazepam (11\%).

\section{ON-STUDY ATTACK CHARACTERISTICS AND TREATMENT}

During the study, 98 (88\%) patients experienced a total of 483 attacks. Of these, 371 (77\%) required treatment at a health care facility and/or hemin administration, with a higher frequency in patients with AIP (79\%) than patients with HCP and VP (44\%; Table 2). The most common treatment location was an inpatient ward (33\%), followed by home (31\%). Hemin treatment was used in $69 \%$ of attacks and opioids were used in $54 \%$ of attacks (Table 2). The median AAR for attacks requiring treatment at a health care facility and/or hemin administration was 2.0 (0.0-37.0). The median AAR was similar for patients with AIP versus patients with HCP or VP (Table 3). The AAR for attacks requiring treatment at a health care facility and/or hemin administration was similar for patients who completed 6 months $(n=107)$ and those who completed the optional extension to 12 months $(\mathrm{n}=80)$. The AAR for attacks requiring treatment at a health care facility and/or hemin administration was also similar whether patients were in the United States or Europe, receiving or not receiving hemin prophylaxis at study entry or on study, or reporting or not reporting chronic daily AHP symptoms at study entry (Table 3). Similar trends were observed for all on-study attacks (including attacks treated at home without hemin), with a median (range) AAR of $3.4(0.0-37.0)$ in patients with AIP, 3.3 (1.5-6.0) in patients with HCP and VP, and 3.4 (0.0-37.0) attacks in all patients (Supporting Table S3). The mean (SD) duration of attacks requiring treatment at a health care facility and/or hemin administration was 7.3 (6.0) days and was higher in patients with HCP and VP than patients with AIP (Table 3).

\section{BASELINE AND ON-STUDY BIOMARKER ASSESSMENTS}

The nonattack mean (SD) urine ALA levels (all samples collected excluding those taken during an

TABLE 2. Characteristics of Attack Treatment During EXPLORE

\begin{tabular}{|c|c|c|c|}
\hline Variable & $\operatorname{AIP}(n=104)$ & VP and HCP $(n=8)$ & Total $(n=112)$ \\
\hline Total attacks & 458 & 25 & 483 \\
\hline Requiring hemin or treatment at a health care facility & $360(79)$ & $11(44)$ & $371(77)$ \\
\hline \multicolumn{4}{|l|}{ Attacks by treatment location } \\
\hline Home & $133(29)$ & $15(60)$ & $148(31)$ \\
\hline Health care facility & $324(71)$ & $10(40)$ & $334(69)$ \\
\hline Inpatient ward & $156(34)$ & $5(20)$ & $161(33)$ \\
\hline Infusion center & $62(14)$ & $2(8)$ & $64(13)$ \\
\hline Clinic office & $58(13)$ & $3(12)$ & $61(13)$ \\
\hline Emergency room & $47(10)$ & $0(0)$ & $47(10)$ \\
\hline Unknown & $1(<1)$ & $0(0)$ & $1(<1)$ \\
\hline \multicolumn{4}{|l|}{ Treatment type } \\
\hline Hemin & $323(71)$ & $9(36)$ & $332(69)$ \\
\hline Opioids & $250(55)$ & $12(48)$ & $262(54)$ \\
\hline Other (carbohydrates, NSAIDs, other) & $202(44)$ & $15(60)$ & $217(45)$ \\
\hline
\end{tabular}

Note: Data are presented as $\mathrm{n}(\%)$.

Abbreviation: NSAID, nonsteroidal anti-inflammatory drug. 
TABLE 3. Characteristics of Attacks Requiring Treatment at a Health Care Facility and/or Hemin Administration Experienced During EXPLORE

\begin{tabular}{|c|c|c|c|}
\hline Variable & $\operatorname{AIP}(n=104)$ & $\mathrm{HCP}$ and VP $(\mathrm{n}=8)$ & Total $(n=112)$ \\
\hline Number of attacks & $2(0-37)$ & $1(0-4)$ & $2(0-37)$ \\
\hline Attack duration per subject (days) & $6.7(3.4)$ & $15.1(18.1)$ & $7.3(6.0)$ \\
\hline AAR & $2.0(0.0-37.0)$ & $1.3(0.0-4.2)$ & $2.0(0.0-37.0)$ \\
\hline \multicolumn{4}{|l|}{ AAR by region } \\
\hline Europe & $1.0(0-37.0)$ & $1.2(1.0-1.5)$ & $1.0(0-37)$ \\
\hline US & $3.3(0.0-15.9)$ & $1.5(0.0-4.2)$ & $2.1(0.0-15.9)$ \\
\hline \multicolumn{4}{|l|}{ AAR by hemin prophylaxis* } \\
\hline Yes & $2.5(0.0-20.0)$ & $3.1(1.9-4.2)$ & $2.5(0.0-20.0)$ \\
\hline No & $2.0(0.0-37.0)$ & $1.0(0.0-2.0)$ & $1.5(0.0-37.0)$ \\
\hline \multicolumn{4}{|l|}{ AAR by daily symptoms ${ }^{\dagger}$} \\
\hline Yes & $2.0(0.0-22.3)$ & $1.0(0.0-4.2)$ & $2.0(0.0-22.3)$ \\
\hline No & $2.8(0.0-37.0)$ & $1.9(1.0-2.0)$ & $2.0(0.0-37.0)$ \\
\hline
\end{tabular}

Note: Data are presented as median (range) or mean (SD). AAR is annualized attack rate, calculated (number of attacks $\div$ time on study). *At study entry or on study.

${ }^{\dagger}$ At study entry.

attack or when affected by hemin treatment) when they were not experiencing attacks (baseline and during study period) were $14.1(9.5) \mathrm{mmol} / \mathrm{mol}$ creatinine in patients with AIP and $3.6(3.4) \mathrm{mmol} / \mathrm{mol}$ creatinine in patients with HCP and VP (although there were only 8 patients with HCP or VP; Supporting Fig. S2A). These values compare with an upper limit of normal (ULN) of $1.5 \mathrm{mmol} / \mathrm{mol}$ creatinine for urinary ALA in healthy non-AHP individuals as determined by LC-MS/MS assay (see Study Assessments). ${ }^{(35)}$ The nonattack mean (SD) urine PBG levels were 32.1 (18.6) $\mathrm{mmol} / \mathrm{mol}$ creatinine in patients with AIP and $1.6(2.4) \mathrm{mmol} / \mathrm{mol}$ creatinine in patients with HCP and VP (Supporting Fig. S2B). These values compare with healthy nonAHP individuals in whom the ULN for urinary PBG is $0.14 \mathrm{mmol} / \mathrm{mol}$ creatinine as determined by LC-MS/ MS assay (see Study Assessments). ${ }^{(35)}$

During attacks on study (any attack type), the maximum urine ALA and PBG levels increased by an average of $193 \%$ and $146 \%$, respectively, compared with the nonattack mean (Fig. $2 \mathrm{~A}$ and $2 \mathrm{~B}$ ). For attacks that required treatment at a health care facility or with hemin, mean peak urine ALA and PBG values were slightly higher compared with other attack types (220\% and 164\%, respectively, for all patients). Following treatment with hemin, mean ALA and PBG levels decreased to near or below levels recorded between attacks.

At baseline (measured in all patients, one sample per patient), normalized urine ALAS1 mRNA levels were approximately 4-fold above levels in normal healthy individuals and approximately 2-fold above levels in chronic high-excreter patients (patients with AHP mutations and elevated urinary ALA/PBG levels but not experiencing acute attacks), as reported in the literature. ${ }^{(36)}$ Baseline normalized ALAS1 mRNA levels were approximately 2-fold higher in patients with AIP than in patients with HCP and VP (Supporting Fig. S2C). During an attack, urine ALAS1 mRNA levels increased by approximately $33 \%$ above the nonattack mean (Fig. 2C). Following treatment with hemin, mean ALAS1 mRNA levels decreased to $27 \%$ below the nonattack mean.

\section{ON-STUDY CHANGES IN HEALTH STATUS}

During the study, 96 (86\%) patients experienced at least one change in health status, and 6\% experienced at least one adverse event (change in health status related to study procedure, e.g., vessel puncture site bruise); all were mild or moderate in severity and none led to study discontinuation. No serious adverse events occurred. The most common on-study changes in health status, as reported by investigators, were headache in 15 (13\%) patients, nasopharyngitis in $14(13 \%)$ patients, influenza and systemic arterial hypertension in $9(8 \%)$ patients, gastroenteritis and arthralgia in $8(7 \%)$ patients, and fatigue, pyrexia, and 

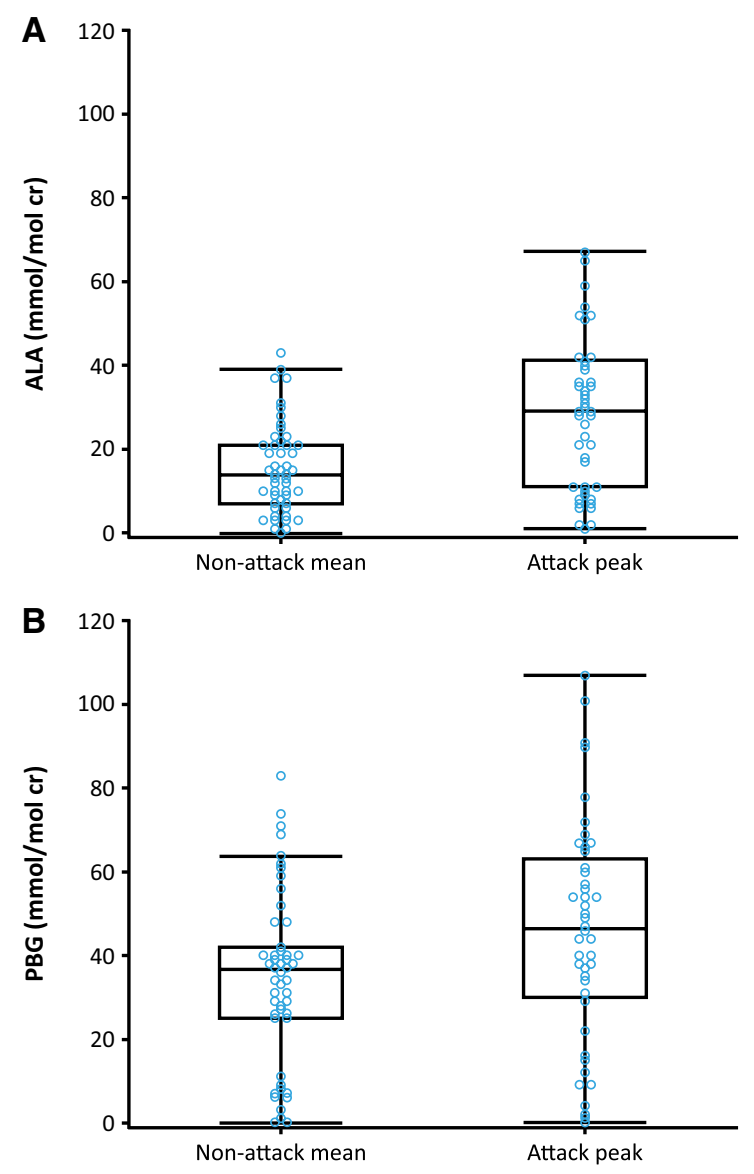

\section{BASELINE AND ON-STUDY LABORATORY ASSESSMENTS}

Mean values for liver function tests, hematology, serum chemistry, and renal parameters remained relatively constant over the course of the study. At baseline, approximately $16 \%$ of all patients had elevations of liver aminotransferases ( $>1 \times \mathrm{ULN}$ and $<3 \times \mathrm{ULN})$. One patient had an increase in alanine aminotransferase and aspartate aminotransferase from $\leq 3 \times \mathrm{ULN}$ at study entry to between $>5 \times$ ULN and $\leq 10 \times \mathrm{ULN}$ at the Month 12 visit. The cause of this elevation was not reported. The patient did not experience an AHP attack at this time and had not had any changes in concomitant medications at or shortly before the time of the elevations. Seventy-six (68\%) patients had reduced eGFR $\left(<90 \mathrm{~mL} / \mathrm{min} / 1.73 \mathrm{~m}^{2}\right)$ during the study, including 31 (28\%) patients who met the criteria for stage $3 \mathrm{a}, 3 \mathrm{~b}$, or $4 \mathrm{CKD},{ }^{(37)}$ based on an eGFR level of $15-59 \mathrm{~mL} / \mathrm{min} / 1.73 \mathrm{~m}^{2}$. ${ }^{(38)}$

\section{BASELINE AND ON-STUDY QoL}

Overall, patients with AHP experiencing recurrent attacks had low $\mathrm{QLL}$ at baseline and throughout the study. At baseline, mean (SD) EQ-5D-5L Index Scores were $0.78(0.15)$ in a patient population with a median age of 38 years, which is lower than the European population norm of 0.92 for EQ-5D-5L (for the 35-44 age group). ${ }^{(39)}$ Self-rated health status using a mean (SD) EQ-5D-5L visual analog scale was decreased to 66 (100 signifying maximal score and best health) at baseline and remained stable over the course of the study. Visual analog scale scores were similar whether or not a patient had ever used prophylactic hemin. The three dimensions most affected 


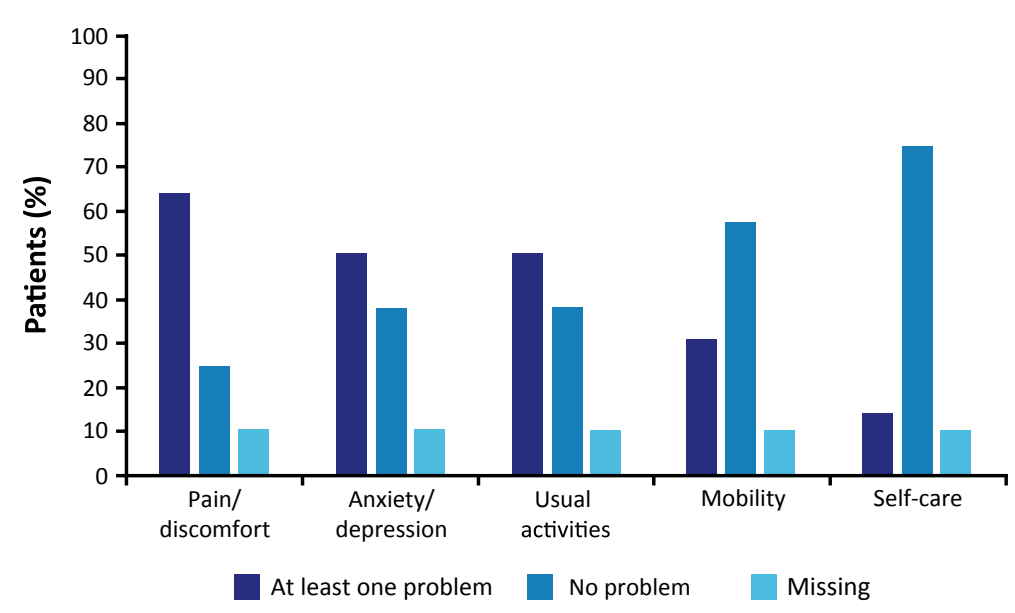

FIG. 3. Patients reporting problems affecting QoL across the five dimensions of EQ-5D-5L.

by AHP, with at least some problems noted, were pain and discomfort (72 [64\%] patients), anxiety and depression (57 [51\%] patients), and ability to perform usual activities (57 [51\%] patients; Fig. 3).

\section{BASELINE AND ON-STUDY HEALTH CARE UTILIZATION AND ECONOMIC IMPACT}

Overall, patients with AHP reported a high level of health care utilization during EXPLORE. At 12 months, patients reported a mean (SD) of 3.6 (8.6) emergency room visits and 5.1 (14.9) overnight hospital stays while on study. Health care utilization was similar regardless of whether or not patients had ever been treated with prophylactic hemin. AHP also had a significant economic impact on patients. Sixty-eight percent of patients were not in full-time work at Month 12; of those who were employed part time or full time, $52 \%$ reported missing many days of work in the last 12 months because of their porphyria (mean [SD] 40.2 [53.9] days missed). Approximately one fifth $(21 \%)$ of patients had received disability payments because of AHP in the past 12 months during the study, with levels similar between US and European patients.

\section{Discussion}

EXPLORE is a multinational, prospective, observational study designed to evaluate the disease activity and clinical management of severely affected patients with AHP who experience recurrent attacks or are on treatment to prevent attacks. Such information is central to understanding the disease manifestations, as well as assessing the need for and evaluation of the benefits and risks of potential new therapies. The study population had a history of recurrent attacks (defined in this study as $\geq 3$ attacks per year) or were on prophylactic treatment to prevent attacks. There was a wide range in the number of attacks experienced by patients, with approximately one third reporting $>10$ attacks in the 12 months before enrollment. Consistent with the literature, symptoms commonly experienced during attacks included pain, nausea, muscle weakness, and tiredness. ${ }^{(12,13)}$ Unique to this study was the high percentage of patients reporting a change in urine color, trouble sleeping, and problems with mental function (potentially because of effects on the central nervous system) during attacks.

A substantial number of patients (approximately two thirds) reported chronic AHP symptoms between attacks, with almost half reporting these symptoms daily. This number is considerably higher than reported in other observational studies (ranging from $18 \%$ to $22 \%)^{(13,14)}$ but likely reflects the more severely affected patient population in this study and that some of the questionnaires were completed directly by the patients. AAR was similar whether patients reported chronic daily symptoms or not, suggesting this is not a strong predictor of attack frequency. Chronic symptoms were also reported to a similar extent whether or not a patient was on hemin prophylaxis. 
All countries in the study, except Bulgaria, had hemin available for attack treatment, with $84 \%$ of the patients reporting prior hemin treatment for an attack. As prophylactic use of hemin is off-label, access can vary by region. In this patient group, treatment practices observed with hemin prophylaxis were similar across geographic regions, although a somewhat higher rate of hemin prophylaxis was noted in the United States than in Europe. However, AAR was similar in the United States and Europe and between patients receiving or not receiving hemin prophylaxis on study or at study entry in this patient group. Consistent with the literature, approximately half of the study population were taking opioid analgesics at study entry during and/or between attacks, supporting the data indicating that the disease has chronic manifestations requiring further treatment. ${ }^{(26)}$

The most common comorbid conditions reported in the medical history at baseline were consistent with the known natural history of patients with AHP. ${ }^{(13,40)}$ Although renal disorders were reported in $10 \%$ of patients in the medical history, approximately $70 \%$ had an abnormal eGFR $\left(<90 \mathrm{~mL} / \mathrm{min} / 1.73 \mathrm{~m}^{2}\right)$, and $30 \%$ met the criteria for stage $3 \mathrm{a}, 3 \mathrm{~b}$, or $4 \mathrm{CKD}$. CKD is reported to impact $30 \%-60 \%$ of patients with AHP and may be due to the toxic effects of chronic, high levels of ALA and PBG on the kidney and influenced by genetic variation in the ALA transporter peptide transporter $2 .(13,20,40,41)$

As has been observed in other studies, disease activity in patients with AIP was detectable through increased biomarker levels during and between attacks. ${ }^{(31,42)}$ At baseline, there were marked elevations in levels of urinary ALA and PBG compared with the ULN in healthy individuals ${ }^{(35)}$ and in $A L A S 1$ mRNA compared with normal levels. ${ }^{(36)}$ Although patients with HCP or VP had near-normal ALA and PBG levels at baseline, in all patients, ALA, PBG, and ALAS1 mRNA levels increased during attacks. This increase suggests an important pathogenetic link of up-regulation of hepatic ALAS1 to disease activity, although differences in ALA, PBG, and ALAS1 mRNA levels were not analyzed for statistical significance. This has been inferred from preclinical studies in cell culture and animals, inferred from liver samples obtained from patients, and recently demonstrated in a clinical study in patients with AIP who were treated with an investigational RNA interference therapeutic givosiran targeting hepatic ALAS1 mRNA. ${ }^{(43-46)}$
An association between elevated ALA and PBG levels and disease activity in patients with AIP has been reported in the literature. ${ }^{(31)}$ Similarly, therapeutics that lower elevated ALA and PBG levels transiently (hemin), in a more prolonged manner (givosiran), and permanently (liver transplantation) have been shown to reduce disease activity. ${ }^{(30,46,47)}$ In EXPLORE, hemin treatment led ALA, PBG, and ALAS1 mRNA levels to decrease to near or below nonattack levels. ALAS1 mRNA up-regulation and increased ALAS1 enzyme activity have been demonstrated in animal models of AIP and in liver tissue from patients with refractory AIP. However, longitudinal data on changes in hepatic ALAS1 mRNA or ALAS1 protein levels have not been previously available because of the requirement for repeated invasive liver biopsies. ${ }^{(43)} \mathrm{By}$ measuring circulating $A L A S 1$ mRNA from exosomes isolated from urine (methodology used in this study), the study confirmed the central importance of hepatic ALAS1 induction in AHP pathophysiology and provided a noninvasive way to measure dynamic changes in hepatic ALAS1, which potentially could serve as a marker of disease activity and therapeutic response in these patients.

At study entry, patients reported diminished QoL while not experiencing an attack compared with population norms, which remained relatively stable at the 6-month and 12-month assessments and did not differ based on prophylactic hemin usage. These data are consistent with other studies that used EQ-5D-5L to demonstrate diminished $\mathrm{QoL}$ in patients with AHP. ${ }^{(15,48)}$ There was also a significant economic burden highlighted in patients from EXPLORE who exhibited increased health care utilization and substantial numbers of lost work days, with over two thirds of patients not in full-time employment and around one quarter receiving disability payments.

A potential limitation of the study is that attacks were patient reported and did not require confirmation by a clinician or by increases in ALA and PBG levels. This method of attack ascertainment, however, largely reflects what occurs in clinical practice after patients have already been diagnosed with AHP, as patients may not be seen by the porphyria specialist for every attack, and ALA and PBG are often not obtained or available in real time when a patient presents with a potential porphyria attack. As it is possible that patients could experience symptoms that were similar to those of attacks but not due to AHP, this 
may have led to an overestimation of attack frequency. However, when measured once an attack was reported, mean ALA and PBG levels increased from baseline, suggesting patient-reported attacks were likely to be related to AHP. In addition, the attack rate during the study actually decreased compared with the attack rates reported by both the investigators and the patients in the year before the study. Finally, over three quarters of attacks required treatment at a health care facility and/or hemin administration, indicating these were serious medical events indicative of AHP attacks.

This prospective study of patients with AHP experiencing recurrent attacks highlights the high level of disease burden in this group and demonstrates that chronic symptoms are present in a majority of such patients between attacks, diminishing their QoL. Overall, data from the EXPLORE study showed that attacks are serious and morbid events, with $>75 \%$ of attacks requiring urgent medical treatment in a hospital or health care facility and/or treatment with intravenous hemin. The use of hemin prophylaxis was reported by nearly half of patients at study entry, whereas more than half received opioid analgesics. Approximately two thirds of patients in EXPLORE experienced chronic symptoms between attacks, with almost half reporting symptoms daily.

Elevated levels of ALA, PBG, and ALAS1 mRNA were observed in patients with AIP outside of attacks and increased further during attacks. In addition, the disease adversely affected multiple aspects of QoL, increased health care utilization, and had a substantial economic impact. These results underscore the need for novel therapies that can reduce attacks and disease manifestations between attacks and improve patients' ability to function on a daily basis.

\section{REFERENCES}

1) Bissell DM, Anderson KE, Bonkovsky HL. Porphyria. N Engl J Med 2017;377:862-872.

2) Puy H, Gouya L, Deybach JC. Porphyrias. Lancet 2010;375: 924-937.

3) Ramanujam VMS, Anderson KE. Porphyria diagnostics-part 1: a brief overview of the porphyrias. Curr Protoc Hum Genet 2015;86:17.20.1-17.20.26.

4) Balwani M, Wang B, Anderson KE, Bloomer JR, Bissell DM, Bonkovsky HL, et al.; Porphyrias Consortium of the Rare Diseases Clinical Research Network. Acute hepatic porphyrias: recommendations for evaluation and long-term management. Hepatology 2017;66:1314-1322.

5) Chen B, Solis-Villa C, Hakenberg J, Qiao W, Srinivasan RR, Yasuda M, et al. Acute intermittent porphyria: predicted pathogenicity of HMBS variants indicates extremely low penetrance of the autosomal dominant disease. Hum Mutat 2016;37:1215-1222.

6) Lenglet $H$, Schmitt $C$, Grange $T$, Manceau $H$, Karboul $N$, Bouchet-Crivat F, et al. From a dominant to an oligogenic model of inheritance with environmental modifiers in acute intermittent porphyria. Hum Mol Genet 2018;27:1164-1173.

7) Stein PE, Badminton MN, Rees DC. Update review of the acute porphyrias. Br J Haematol 2017;176:527-538.

8) Bissell DM, Lai JC, Meister RK, Blanc PD. Role of delta-aminolevulinic acid in the symptoms of acute porphyria. Am J Med 2015;128:313-317.

9) Anderson KE, Bloomer JR, Bonkovsky HL, Kushner JP, Pierach CA, Pimstone NR, et al. Recommendations for the diagnosis and treatment of the acute porphyrias. Ann Intern Med 2005;142:439-450.

10) Elder G, Harper P, Badminton M, Sandberg S, Deybach JC. The incidence of inherited porphyrias in Europe. J Inherit Metab Dis 2013;36:849-857.

11) Schmitt C, Lenglet H, Yu A, Delaby C, Benecke A, Lefebvre $\mathrm{T}$, et al. Recurrent attacks of acute hepatic porphyria: major role of the chronic inflammatory response in the liver. J Intern Med 2018;284:78-91.

12) Simon A, Pompilus F, Querbes W, Wei A, Strzok S, Penz C, et al. Patient perspective on acute intermittent porphyria with frequent attacks: a disease with intermittent and chronic manifestations. Patient 2018;11:527-537.

13) Bonkovsky HL, Maddukuri VC, Yazici C, Anderson KE, Bissell $\mathrm{DM}$, Bloomer JR, et al. Acute porphyrias in the USA: features of 108 subjects from porphyrias consortium. Am J Med 2014;127:1233-1241.

14) Andersson $C$, Innala $E$, Bäckström $T$. Acute intermittent porphyria in women: clinical expression, use and experience of exogenous sex hormones. A population-based study in northern Sweden. J Intern Med 2003;254:176-183.

15) Millward LM, Kelly P, Deacon A, Senior V, Peters TJ. Self-rated psychosocial consequences and quality of life in the acute porphyrias. J Inherit Metab Dis 2001;24:733-747.

16) Bjersing L, Andersson C, Lithner F. Hepatocellular carcinoma in patients from northern Sweden with acute intermittent porphyria: morphology and mutations. Cancer Epidemiol Biomarkers Prev 1996;5:393-397.

17) Andersson C, Bjersing L, Lithner F. The epidemiology of hepatocellular carcinoma in patients with acute intermittent porphyria. J Intern Med 1996;240:195-201.

18) Pischik E, Kauppinen R. An update of clinical management of acute intermittent porphyria. Appl Clin Genet 2015;8:201-214.

19) Peoc'h K, Manceau H, Karim Z, Wahlin S, Gouya L, Puy H, et al. Hepatocellular carcinoma in acute hepatic porphyrias: a Damocles Sword. Mol Genet Metab 2018. doi:https://doi.org/10.1016/ j.ymgme.2018.10.001. In press.

20) Pallet N, Mami I, Schmitt C, Karim Z, François A, Rabant M, et al. High prevalence of and potential mechanisms for chronic kidney disease in patients with acute intermittent porphyria. Kidney Int 2015;88:386-395.

21) Balwani M. What hematologists need to know about acute hepatic porphyria. Clin Adv Hematol Oncol 2016;14:858-861.

22) Stein PE, Badminton MN, Barth JH, Rees DC, Sarkany R, Stewart MF, et al. Acute intermittent porphyria: fatal complications of treatment. Clin Med (Lond) 2012;12:293-294.

23) Orphan Europe. Summary of product characteristics: Normosang $25 \mathrm{mg} / \mathrm{ml}$, concentrate for solution for infusion. Published June 2007. https://www.mhlw.go.jp/shingi/2008/03/d1/s0326-10n.pdf. Accessed September 19, 2019.

24) Recordati Rare Diseases Inc. US prescribing information: PANHEMATIN (hemin for injection). Published 2017. 
https://www.panhematin.com/pdf/panhematin-marketing-PI.pdf. Accessed September 19, 2019.

25) von und zu Fraunberg M, Pischik E, Udd L, Kauppinen R. Clinical and biochemical characteristics and genotype-phenotype correlation in 143 Finnish and Russian patients with acute intermittent porphyria. Medicine (Baltimore) 2005;84:35-47.

26) Marsden JT, Guppy S, Stein P, Cox TM, Badminton M, Gardiner $\mathrm{T}$, et al. Audit of the use of regular haem arginate infusions in patients with acute porphyria to prevent recurrent symptoms. JIMD Rep 2015;22:57-65.

27) Innala E, Bäckström T, Bixo M, Andersson C. Evaluation of gonadotropin-releasing hormone agonist treatment for prevention of menstrual-related attacks in acute porphyria. Acta Obstet Gynecol Scand 2010;89:95-100.

28) Schulenburg-Brand D, Gardiner T, Guppy S, Rees DC, Stein P, Barth J, et al. An audit of the use of gonadorelin analogues to prevent recurrent acute symptoms in patients with acute porphyria in the United Kingdom. JIMD Rep 2017;36:99-107.

29) Kauppinen R, Mustajoki P. Prognosis of acute porphyria: occurrence of acute attacks, precipitating factors, and associated diseases. Medicine (Baltimore) 1992;71:1-13.

30) Dowman JK, Gunson BK, Mirza DF, Bramhall SR, Badminton MN, Newsome PN; UK Liver Selection and Allocation Working Party. Liver transplantation for acute intermittent porphyria is complicated by a high rate of hepatic artery thrombosis. Liver Transpl 2012;18:195-200.

31) Kauppinen R, von und zu Fraunberg M. Molecular and biochemical studies of acute intermittent porphyria in 196 patients and their families. Clin Chem 2002;48:1891-1900.

32) Neeleman RA, Wagenmakers MAEM, Koole-Lesuis RH, Mijnhout GS, Wilson JHP, Friesema ECH, et al. Medical and financial burden of acute intermittent porphyria. J Inherit Metab Dis 2018;41:809-817.

33) Janssen MF, Pickard AS, Golicki D, Gudex C, Niewada M, Scalone $\mathrm{L}$, et al. Measurement properties of the EQ-5D-5L compared to the EQ-5D-3L across eight patient groups: a multi-country study. Qual Life Res 2013;22:1717-1727.

34) Zhang J, Yasuda M, Desnick RJ, Balwani M, Bishop D, Yu C. A LC-MS/MS method for the specific, sensitive, and simultaneous quantification of 5-aminolevulinic acid and porphobilinogen.J Chromatogr B Analyt Technol Biomed Life Sci 2011;879:2389-2396.

35) Anderson KE, Bissell DM, Bonkovsky H, Sardh E, Harper P, Balwani $\mathrm{M}$, et al. Phase 1, 2 and open label extension studies of givosiran, an investigational RNA interference (RNAi) therapeutic, in patients with acute intermittent porphyria. Presented at: The Liver Meeting 2018 of the American Association for the Study of Liver Diseases (AASLD); November 9-13, 2018; San Francisco, CA.

36) Chan A, Liebow A, Yasuda M, Gan L, Racie T, Maier M, et al. Preclinical development of a subcutaneous ALAS1 RNAi therapeutic for treatment of hepatic porphyrias using circulating RNA quantification. Mol Ther Nucleic Acids 2015;4:e263.

37) National Kidney Foundation. K/DOQI clinical practice guidelines for chronic kidney disease: evaluation, classification, and stratification. Am J Kidney Dis 2002;39:S1-S266.

38) Webster AC, Nagler EV, Morton RL, Masson P. Chronic kidney disease. Lancet 2017;389:1238-1252.

39) Janssen B, Szende A. Population norms for the EQ-5D. In: Szende A, Janssen B, Cabases J, eds. Self-Reported Population Health: An International Perspective Based on EQ-5D. Dordrecht, the Netherlands: Springer; 2014:19-30.

40) Pallet N, Karras A, Thervet E, Gouya L, Karim Z, Puy H. Porphyria and kidney diseases. Clin Kidney J 2018;11:191-197.

41) Tchernitchko D, Tavernier Q, Lamoril J, Schmitt C, Talbi N, Lyoumi S, et al. A variant of peptide transporter 2 predicts the severity of porphyria-associated kidney disease. J Am Soc Nephrol 2017;28:1924-1932.

42) Marsden JT, Rees DC. Urinary excretion of porphyrins, porphobilinogen and $\delta$-aminolaevulinic acid following an attack of acute intermittent porphyria. J Clin Pathol 2014;67:60-65.

43) Yasuda M, Gan L, Chen B, Kadirvel S, Yu C, Phillips JD, et al. RNAi-mediated silencing of hepatic Alas1 effectively prevents and treats the induced acute attacks in acute intermittent porphyria mice. Proc Natl Acad Sci USA 2014;111:7777-7782.

44) Yasuda M, Erwin AL, Liu LU, Balwani M, Chen B, Kadirvel S, et al. Liver transplantation for acute intermittent porphyria: biochemical and pathologic studies of the explanted liver. Mol Med 2015;21:487-495.

45) Zheng J, Shan Y, Lambrecht RW, Donohue SE, Bonkovsky HL. Differential regulation of human ALAS1 mRNA and protein levels by heme and cobalt protoporphyrin. Mol Cell Biochem 2008;319:153-161.

46) Sardh E, Harper P, Balwani M, Stein P, Rees D, Bissell DM, et al. Phase 1 trial of an RNA interference therapy for acute intermittent porphyria. N Engl J Med 2019;380:549-558.

47) Sardh E, Harper P, Andersson DE, Floderus Y. Plasma porphobilinogen as a sensitive biomarker to monitor the clinical and therapeutic course of acute intermittent porphyria attacks. Eur J Intern Med 2009;20:201-207.

48) Jiménez-Monreal AM, Murcia MA, Gómez-Murcia V, Bibiloni Mdel M, Pons A, Tur JA, et al. Anthropometric and quality-of-life parameters in acute intermittent porphyria patients. Medicine (Baltimore) 2015;94:e1023.

\section{Supporting Information}

Additional Supporting Information may be found at onlinelibrary.wiley.com/doi/10.1002/hep.30936/suppinfo. 